\title{
BOEMDET-Band Offsets and Effective Mass Determination Technique utilizing Fowler-Nordheim tunneling slope constants in MIS devices on silicon.
}

\author{
Ravi Kumar Chanana \\ Department of Electrical and Electronics Engineering, Galgotia's College of Engineering and Technology, \\ Affiliated to Uttar Pradesh Technical University, Lucknow, India. \\ 1, Institutional Area, Knowledge Park-II, Greater Noida - 201306, India.
}

\begin{abstract}
The Fowler-Nordheim carrier tunneling slope constants for electron and hole conduction through MOS devices fabricated on silicon substrate are utilized to determine the conduction and valence band offsets, carrier effective masses in the $\mathrm{SiO}_{2}$ and its unknown bandgap, independent of photoemission spectroscopic measurements of band offsets on $\mathrm{SiO}_{2} / \mathrm{Si}$ samples. The slope constants are obtained from the electron and hole tunneling currents versus voltage characteristics on a pair of n-MOS and p-MOS devices in accumulation or an n-channel MOSFET device in inversion that allows carrier separation. This characterization technique called BOEMDET, can be applied to other insulating materials grown or deposited on silicon, such as JVD nitride, HfSiON, SiO $N_{x} N_{y}$ and high-K oxides found viable for future MOS technology. To an accuracy of one decimal place, the conduction band offset for the $\mathrm{SiO}_{2} / \mathrm{Si}<100>\mathrm{MIS}$ structure is determined to be $3.2 \mathrm{eV}$, the valence band offset obtained is $4.6 \mathrm{eV}$ and the bandgap of $\mathrm{SiO}_{2}$ is found to be $8.9 \mathrm{eV}$. The electron and hole effective masses in the $\mathrm{SiO}_{2}$ are calculated to be $0.42 \mathrm{~m}$ and $0.58 \mathrm{~m}$, where $\mathrm{m}$ is the free electron mass. In case of quantum confinement at the $\mathrm{SiO}_{2} / \mathrm{Si}$ interface, the electron and hole masses correct to $0.51 \mathrm{~m}$ and $0.65 \mathrm{~m}$. Also, the carrier effective masses in thermal $\mathrm{SiO}_{2}$ are found to be the same for all thickness of the oxide.
\end{abstract}

Keywords: effective mass, FN-tunneling, band offsets, metal-insulator-semiconductor

\section{Introduction}

High dielectric constant oxides are viable candidates for metal-insulator-semiconductor (MIS) devices that can allow thicker layers to limit leakage current in the off state through the devices. The thicker layers can at the same time provide the same insulator capacitance per unit area as that of a thin layer of $\mathrm{SiO}_{2}$ which has a low relative dielectric constant $\mathrm{K}$ of 3.9. The list of the viable oxide materials is provided in two recent reviews $[1,2]$. The reviews describe research on various material issues pertaining to dielectric material for CMOS applications. The conduction through these dielectrics at high fields greater than $8 \mathrm{MV} / \mathrm{cm}$ is described by the Fowler-Nordheim (FN) tunneling mechanism [3-7]. They contain traps in the bulk that causes Poole-Frenkel conduction at intermediate oxide fields of 5-7 MV/cm [8,9]. The traps in the oxide trap electrons under voltage or current stress and shift the threshold voltage of the MOSFET device [10]. There is constant research into making these oxides amorphous and free of bulk traps to make them suitable as electronic materials. This causes reduction in the leakage current by about $10^{4}$ times that in the $\mathrm{SiO}_{2}$ at $1 \mathrm{~V}$ for the same equivalent oxide thickness [2]. The process of making the film amorphous also causes reduction in $\mathrm{K}$ value, increase in the interface trap density, and addition of fixed charges [2]. Two suitable amorphous dielectric materials for CMOS applications are thermal $\mathrm{SiO}_{2}$ and Jet Vapor Deposited (JVD) silicon nitride that exhibit only FN tunneling because of negligible bulk traps $[11,12]$. In a MOS device, FN tunneling mechanism can be utilized to determine the conduction and valence band offsets with $\mathrm{Si}<100>$ surface, the electron and hole effective masses in $\mathrm{SiO}_{2}$, and determination of the unknown bandgap of $\mathrm{SiO}_{2}[6,7]$. This is the subject of the present study, but it has a different perspective. The FN tunneling slope constants are the only measured parameters that are used to determine the above MOS device parameters. The study will facilitate simulation of FN tunneling through a MOS device at high fields, and consequently estimate its breakdown field strength and the onset field for FN tunneling [7]. This FN technique called BOEMDET of characterizing an MIS structure is an alternative option to the photoemission spectroscopic measurements of band offsets and bandgap.

\section{Theory}

FN electron and hole tunneling has been observed in $\mathrm{Si}$ and $\mathrm{SiC}$ MOS devices and in organic light emitting diodes $[3,5,13]$. The FN equation models the current-voltage characteristics across a MOS device at high fields. It is given by the classical equation $[4,5]$ : 


$$
\frac{J}{E^{2}}=A \exp \left(\frac{-B}{E}\right) \ldots \ldots(1)
$$

where $\mathrm{J}$ is the current density across the MOS device in $\mathrm{A} / \mathrm{cm}^{2}, \mathrm{E}$ is the oxide electric field in $\mathrm{V} / \mathrm{cm}$, and the preexponent $\mathrm{A}$ and the slope constant $\mathrm{B}$ are given by:

$$
\begin{aligned}
A & =\frac{e^{3} m}{16 \pi^{2} \hbar m_{o x} \phi_{0}} \ldots . .(2) \\
A & =1.54 \times 10^{-6} \frac{m}{m_{o x}} \frac{1}{\phi_{0}} \ldots \ldots\left(A / V^{2}\right) \\
B & =\frac{4}{3} \frac{\left(2 m_{o x}\right)^{1 / 2}}{e \hbar} \phi_{0}^{3 / 2} \ldots \ldots(3) \\
B & =6.83 \times 10^{7}\left(\frac{m_{o x}}{m}\right)^{1 / 2} \phi_{0}^{3 / 2} \ldots \ldots(V / \mathrm{cm})
\end{aligned}
$$

In $\mathrm{A}$ and $\mathrm{B}$ constants, $\mathrm{e}$ is the electronic charge, $\mathrm{m}$ is the free electron mass, $\mathrm{m}_{\mathrm{ox}}$ is the electron or hole mass in the oxide, $2 \pi \hbar$ is Planck's constant and $\phi_{0}$ is the electron or hole barrier height expressed in electron volts. A plot of $\ln \left(\mathrm{J} / \mathrm{E}^{2}\right)$ versus $1 / \mathrm{E}$, called an $\mathrm{FN}$ plot, gives the value of the slope constant $\mathrm{B}$, from which $\left(m_{o x} / m\right)^{1 / 2} \phi_{0}^{3 / 2}$ product can be obtained. Then, with a known effective mass, $\phi_{0}$ can be calculated, and with a known $\phi_{0}$, the effective mass in the oxide can be calculated. The slope constant B is very sensitive to the oxide field as it is in the exponential and therefore precise determination of the oxide field is absolutely critical in the evaluation of the tunneling parameters. The $\ln \left(\mathrm{J} / \mathrm{E}^{2}\right)$ term is relatively much less sensitive to the oxide field as it is in the natural logarithm. The slope constant B can be independently used to determine the carrier effective masses, band offsets at the insulator-semiconductor surface and the insulator bandgap, without the knowledge of band offsets from photoemission spectroscopic measurements. This however is possible on the silicon substrate, where the grown or deposited dielectric film forms an abrupt interface and the intrinsic Fermi level of silicon lies very near the middle of its bandgap after the growth or deposition of the film due to negligible intrinsic defects in the silicon substrate. The slope constants for electron current $B_{e}$, and for the hole current $B_{h}$, can be obtained from the current-voltage characteristics on either a pair of n-MOS and p-MOS devices in accumulation or an $\mathrm{n}$-channel silicon MOSFET in inversion that allows carrier separation. Due to carrier separation, both the electron and hole currents versus oxide voltage characteristics are obtained from a single MOSFET device.

\section{A. Average oxide voltage in MOS devices}

A MOS device is a two-terminal device and therefore, one dominating current can be measured at a time when the device is biased in accumulation. For an n-MOS device with the semiconductor as cathode, the dominant electron tunneling current can be measured. For a p-MOS device with the semiconductor as the anode, the dominant hole tunneling current can be measured, provided the hole barrier from the silicon valence band to oxide valence band is less than the electron barrier at the metal gate cathode. This can be achieved in silicon p-MOS device by having $\mathrm{p}+$-poly silicon carbide as the gate electrode providing $6 \mathrm{eV}$ valence electron barrier. The hole barrier of $4.6 \mathrm{eV}$ at the anode will then become less to allow the dominant hole tunneling current through the device in accumulation. Thus a pair of n-MOS and p-MOS devices on silicon having metal gates and same thickness oxide can provide electron current and hole current versus oxide voltage characteristics, from which the inter-related FN slope constants $B_{e}$ and $B_{h}$ can be obtained. The figure 1 below shows the n-MOS and p-MOS devices with poly-SiC gates in accumulation. 


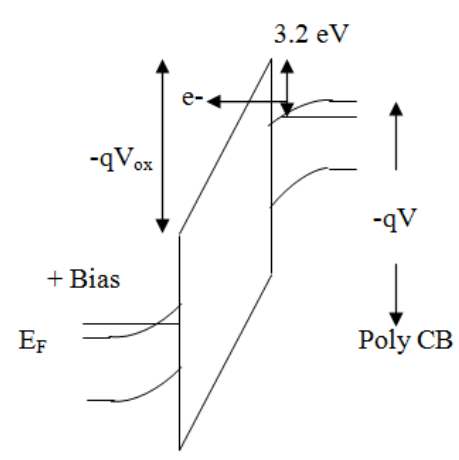

(a)

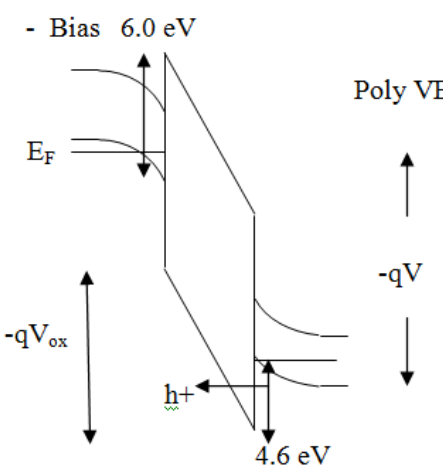

(b)

$n^{+}$Poly-SiC $\quad \mathrm{SiO}_{2} \quad n-\mathrm{Si} \quad \mathrm{p}^{+}$Poly-SiC $\quad \mathrm{SiO}_{2} \quad$ p-Si

Fig. 1 Silicon MOS devices in accumulation: a) n-MOS and b) p-MOS with poly-SiC gates.

The formulation of the average oxide voltage in a pair of n-MOS and p-MOS devices is presented in the author's earlier report [6]. The formulae are presented here again and it is shown that the oxide voltage is corrected by the flatband voltage, $\mathrm{V}_{\mathrm{fb}}$, in MOS devices having thick oxides and metal gates. For a n-MOS device in accumulation and having positive charges or negative $\mathrm{V}_{\mathrm{fb}}$, and electron tunneling as the dominant current, the $\mathrm{V}_{\text {ox }}$ is given as, 1

$$
V_{o x}=\left|V_{a p p}\right|-\left|V_{f b}\right| \ldots . . .(4) \text {; }
$$

where, $\mathrm{V}_{\text {app }}$ is the applied voltage across the device. This is the case in Si technology, where the charges in the gate oxide is generally positive and the metal-semiconductor work function difference, $\mathrm{W}_{\mathrm{ms}}$, exists as part of $\mathrm{V}_{\mathrm{fb}}$ obtained from the capacitance-voltage characteristics [6]. For the oxide with net negative charges or positive $\mathrm{V}_{\mathrm{fb}}$, the oxide voltage is given as:

$V_{o x}=\left|V_{a p p}\right|+\left|V_{f b}\right| \ldots \ldots$ (5) .

In case of hole tunneling as the dominant current in a p-MOS device in accumulation, the oxide having positive charges or negative $\mathrm{V}_{\mathrm{fb}}$ will have $\mathrm{V}_{\mathrm{ox}}$ as:

$V_{o x}=\left|V_{a p p}\right|+\left|V_{f b}\right| \ldots \ldots(6)$,

and the oxide having net negative charges or positive $\mathrm{V}_{\mathrm{fb}}$, the oxide voltage is given as;

$V_{o x}=\left|V_{a p p}\right|-\left|V_{f b}\right| \ldots \ldots$ (7).

B. Average oxide voltage in a n-channel MOSFET device

The corrected oxide voltages for electron and hole conduction through the oxide in an $\mathrm{n}$-channel silicon MOSFET biased in inversion has been formulated in a recent report [7]. It is presented here again for reference. It needs to be noted that the n-channel MOSFET device is a three terminal device and allows carrier separation. The electron tunneling current can be obtained as the gate current and the hole current can be obtained as the substrate current. Thus, from one device, both the electron and hole tunneling current versus oxide voltage characteristics can be obtained as compared to two MOS devices required for the same currents.

$$
V_{o x}=\left|V_{a p p}\right|+\left|V_{s b}\right|-\left|V_{T}\right|+\left|\frac{Q_{d}}{C_{i}}\right| \ldots \ldots \text { (8); }
$$

for the gate electron conduction, and

$$
V_{o x}=\left|V_{a p p}\right|+\left|V_{s b}\right|+\left|V_{T}\right|-\left|\frac{Q_{d}}{C_{i}}\right| \ldots \ldots \text { (9) }
$$

for the substrate hole conduction through the oxide. Here, $V_{\text {app }}$ is the applied gate voltage, $V_{\mathrm{sb}}$ is the substrate reverse bias used for controlling the threshold voltage, $V_{T}$ is the threshold voltage of the device, $Q_{d}$ is the depletion charge in $\mathrm{C} / \mathrm{cm}^{2}$ in the channel, and $\mathrm{C}_{\mathrm{i}}$ is the insulator capacitance in $\mathrm{F} / \mathrm{cm}^{2}$. It can be observed that the oxide voltage for electron conduction is different from the oxide voltage for hole conduction and they are both corrected by the threshold voltage. 
C. Modification of the FN slope constant equations for devices on silicon

It has been observed from a recent report of soft X-ray photoemission experiments on $2 \mathrm{~nm}$ dry thermal $\mathrm{SiO}_{2}$ [14], that the conduction and valence band offsets from the intrinsic silicon Fermi level are $3.8 \mathrm{eV}$ and 5.1 $\mathrm{eV}$ respectively, and the bandgap of $\mathrm{SiO}_{2}$ is $8.9 \mathrm{eV}$ with the error of $\pm 0.1 \mathrm{eV}$. Taking the ratios of these nominal values as 3.8/8.9 and 5.1/8.9 gives the electron and hole effective masses in the $\mathrm{SiO}_{2}$ of $0.427 \mathrm{~m}$ and $0.573 \mathrm{~m}$ respectively. Within the experimental error of $\pm 0.1 \mathrm{eV}$ in the band offset measurements, the masses can be valued as $0.42 \mathrm{~m}$ and $0.58 \mathrm{~m}$ as reported earlier $[6,7]$. This ratio describes the ratio of the photo-emitted electron or hole kinetic energies during photoemission into the oxide conduction and valence bands as $\frac{0.5 m_{o x, e} v^{2}}{0.5\left(m_{o x, e}+m_{o x, h}\right) v^{2}} \quad$ which equals $\frac{m_{o x, e}}{m}$, thus giving the ratio of electron effective mass to the sum of electron and hole effective masses. This sum equals the free electron mass for the insulators and therefore the band offsets to insulator bandgap ratio equals the relative carrier effective masses. Therefore the relative electron and hole effective masses $\frac{m_{o x, e}}{m}$ and $\frac{m_{o x, h}}{m}$ can be written as $\frac{\left(\phi_{e}+0.55\right)}{E_{g}}$ and $\frac{\left(\phi_{h}+0.57\right)}{E_{g}}$ when the insulator is grown or deposited on $\mathrm{Si}<100>$ or $\mathrm{Si}<111>$ surface. Here, $\phi_{e}$ is the electron band offset from silicon conduction band to oxide conduction band and $\phi_{h}$ is the hole band offset from the silicon valence band to insulator valence band and 0.55 and $0.57 \mathrm{eV}$ are added respectively to coincide the band offsets from the intrinsic Fermi level of silicon. The intrinsic Fermi level of silicon is given by $\frac{E_{c}+E_{v}}{2}+\frac{k T}{2} \ln \left[\frac{N_{v}}{N_{c}}\right]$, where $E_{c}$ is the bottom of the conduction band, $E_{v}$ is the top of the valence band, $N_{c}$ is the effective density of states in the conduction band, and $\mathrm{N}_{\mathrm{v}}$ is the effective density of states in the valence band. $\mathrm{N}_{\mathrm{c}}$ for silicon equals $2.8 \times 10^{19} / \mathrm{cm}^{3}$ and $\mathrm{N}_{\mathrm{v}}$ equals $1.04 \times 10^{19} / \mathrm{cm}^{3}$ at $300 \mathrm{~K}$ temperature [15]. Evaluating the above equation gives the position of intrinsic Fermi level of silicon about $0.01 \mathrm{eV}$ above the middle of the silicon bandgap. Thus $0.55 \mathrm{eV}$ is added to $\phi_{e}$ and $0.57 \mathrm{eV}$ is added to $\phi_{h}$ for the conduction and valence band offsets from the intrinsic Fermi level of silicon. If $B_{e}$ and $B_{h}$ are the electron and hole tunneling slope constants, then the slope constant equations can be written as below:

$$
\begin{aligned}
& B_{e}=6.83 \times 10^{7}\left(\frac{m_{o x, e}}{m}\right)^{1 / 2} \phi_{e}^{3 / 2} \ldots(10) \\
& B_{h}=6.83 \times 10^{7}\left(\frac{m_{o x, h}}{m}\right)^{1 / 2} \phi_{h}^{3 / 2} \ldots(11) \\
& \text { Substituting for } \frac{m_{o x, e}}{m} \text { and } \frac{m_{o x, h}}{m}, \text { the equations become } \\
& B_{e}=6.83 \times 10^{7}\left(\frac{\phi_{e}+0.55}{E_{g}}\right)^{1 / 2} \phi_{e}{ }^{3 / 2} \ldots(12) \\
& B_{h}=6.83 \times 10^{7}\left(\frac{\phi_{h}+0.57}{E_{g}}\right)^{1 / 2} \phi_{h}{ }^{3 / 2} \ldots(13) \\
& E_{g}=\phi_{e}+\phi_{h}+1.12 \ldots . .(14)
\end{aligned}
$$

The equations (12), (13), and (14) form three non-linear simultaneous equations with three unknowns that can be solved with a simple MATLAB software program or by trial and error for a given $B_{e}$ and $B_{h}$ values $[6,7]$. This evaluation will result in the values of $\phi_{e}, \phi_{h}$ and $\mathrm{E}_{\mathrm{g}}$. Following this evaluation, the band offsets from the intrinsic silicon Fermi level can be obtained. Further, the carrier effective masses and the unknown bandgap of the insulator can be determined. The character of an MIS structure can thus be described by the below mentioned one row table as: 


\begin{tabular}{|l|l|l|l|l|}
\hline$\phi_{e}$ & $\phi_{h}$ & $E_{g}$ & $m_{e}$ & $m_{h}$ \\
\hline
\end{tabular}

\section{Calculations and Results:}

A calculation for the FN electron and hole tunneling slope constants, $B_{e}$ and $B_{h}$, was performed utilizing the current-voltage data on $\mathrm{an}^{+} \mathrm{n}^{+}$-polysilicon gated $\mathrm{n}$-channel Si MOSFET device [16] in a recent report [7]. The calculated slope constants for the MOSFET device having an $8.5 \mathrm{~nm}$ dry thermal $\mathrm{SiO}_{2}$, are presented again below for reference and calculations.

Table I. Electron an hole tunneling slope constants $\mathrm{B}_{\mathrm{e}}$ and $\mathrm{B}_{\mathrm{h}}$ for the $\mathrm{n}$-channel MOSFET device

\begin{tabular}{|l|l|l|}
\hline Current type & $\mathrm{B}_{\mathrm{e}}(\mathrm{MV} / \mathrm{cm})$ & $\mathrm{B}_{\mathrm{h}}(\mathrm{MV} / \mathrm{cm})$ \\
\hline $\begin{array}{l}\text { Gate tunneling electron current from } \\
\text { cathode }\end{array}$ & \multicolumn{1}{|c|}{ 254 } & 514 (More Precisely) \\
\hline $\begin{array}{l}\text { Substrate hole current over the anode } \\
\text { barrier }\end{array}$ & ------ & \\
\hline
\end{tabular}

These values are substituted in equations (12) and (13). The equations (12), (13), and (14) are then solved simultaneously giving the values of the MOS device parameters as follows:

$$
\phi_{e}=3.204216 \mathrm{eV} ; \quad \phi_{h}=4.605939 \mathrm{eV} ; \quad \mathrm{E}_{\mathrm{g}}=8.930156 \mathrm{eV} \text {. }
$$

These values are rounded off to three decimal places to give:

$\phi_{e}=3.204 \mathrm{eV} ; \phi_{h}=4.606 \mathrm{eV} ; \mathrm{E}_{\mathrm{g}}=8.930 \mathrm{eV}$.

Further calculations give the electron and hole effective masses in the $\mathrm{SiO}_{2}$ as $0.42 \mathrm{~m}$ and $0.58 \mathrm{~m}$ for free Fermi gas model of carriers at the emitting electrode. Thus, a unique set of conduction and valence band offset values, the unknown bandgap of $\mathrm{SiO}_{2}$ and the effective masses of electrons and holes in $\mathrm{SiO}_{2}$ are determined by this method, only from the knowledge of inter-related FN electron and hole tunneling slope constants $\mathrm{B}_{\mathrm{e}}$ and $\mathrm{B}_{\mathrm{h}}$. These values are presented in Table II below. The electron and hole effective masses in the different high-K oxides grown by the atomic layer deposition (ALD) method, and which are viable for the future MOS technology are also calculated and presented in Table II below. These values are based on the photoemission spectroscopic measurements presented in reference [14].

Table II. The electron and hole effective masses in different high-K oxides and thermal $\mathrm{SiO}_{2}$.

\begin{tabular}{|c|c|c|c|c|c|c|}
\hline Oxide [Ref.] & $\begin{array}{l}\text { Growth process } \\
\text { and thickness in } \\
(\mathrm{nm})\end{array}$ & $\begin{array}{l}\Delta \mathrm{E}_{\mathrm{c}} \quad \text { from } \\
\mathrm{E}_{\mathrm{i}}(\mathrm{eV})\end{array}$ & $\begin{array}{l}\Delta E_{V} \text { from } E_{i} \\
(e V)\end{array}$ & $\begin{array}{l}\text { Band gap } \\
E_{g}(e V)\end{array}$ & $\begin{array}{l}\text { Electron effective } \\
\text { mass in oxide } \\
\frac{m_{o x, e}}{m}=\frac{\Delta E_{c}}{E_{g}}\end{array}$ & $\begin{array}{l}\text { Hole effective mass } \\
\text { in } \\
\frac{m_{o x, h}}{m}=\frac{\Delta E_{v}}{E_{g}}\end{array}$ \\
\hline $\mathrm{HfO}_{2}[14]$ & ALD, 1.5 & 2.4 & 3.3 & 5.7 & 0.42 & 0.58 \\
\hline $\mathrm{Hf}_{0.7} \mathrm{Si}_{0.3} \mathrm{O}_{2}[14]$ & ALD, 1.5 & 2.6 & 3.4 & 6.0 & 0.43 & 0.57 \\
\hline $\mathrm{SiO}_{2}[17]$ & $\begin{array}{l}\text { Remote Plasma, } \\
1.0\end{array}$ & - & 5.1 & 8.9 & - & 0.573 \\
\hline $\mathrm{SiO}_{2}[14]$ & Dry thermal,2.0 & 3.8 & 5.1 & 8.9 & 0.427 & 0.573 \\
\hline $\begin{array}{l}\mathrm{SiO}_{2}, \quad \text { [present } \\
\text { work] }\end{array}$ & Dry thermal, 8.5 & 3.754 & 5.176 & 8.930 & 0.42 & 0.58 \\
\hline
\end{tabular}

\section{Discussion}

In the present study, only the $\mathrm{B}_{\mathrm{e}}$ and $\mathrm{B}_{\mathrm{h}}$ values have been utilized to determine the band offsets and $\mathrm{SiO}_{2}$ bandgap, and the electron and hole effective masses of $0.42 \mathrm{~m}$ and $0.58 \mathrm{~m}$. These are presented in Table II. The method employed is different from the previous studies [6,7], where the literature value of the electron effective mass of $0.42 \mathrm{~m}$ was utilized to determine the conduction band offset of $3.2 \mathrm{eV}$ in $\mathrm{SiO}_{2} / \mathrm{Si}<100>$ sample [7], and $2.78 \mathrm{eV}$ in $\mathrm{SiO}_{2} / 4 \mathrm{H}-\mathrm{SiC}<0001>$ sample [6]. Further, the photoemission bandgap measurement of $8.9 \pm 0.1 \mathrm{eV}$ was utilized to determine the hole effective mass in the $\mathrm{SiO}_{2}$ of $0.58 \mathrm{~m} \mathrm{[6,7]}$. However, the presently determined values can be compared to the soft X-ray photoemission measurement results of band offsets and band gap of $\mathrm{SiO}_{2}$ [14]. It can be observed from Table II to be nearly the same. The FN technique based on the I-V/C-V measurements thus acts as an alternative option to the photoemission measurements. Further, since the tunneling distance in FN tunneling does not change appreciably due to Schottky barrier lowering [4], the FN technique can be supported to provide relatively more accurate measurement than the photoemission spectroscopy which has a resolution of $\pm 0.1 \mathrm{eV}$. The FN technique has been demonstrated to 
give an error in the electron effective mass calculations of less than 4\% [4]. Also, the FN technique applied to the determination of valence band offset of $4.606 \mathrm{eV}$ from an n-channel MOSFET device appears to be more accurate because the Schottky barrier lowering which is a source of error in the photoemission spectroscopy measurements does not exist at the anode of the n-channel MOSFET [7].

The high-K oxides $[1,2,14]$ found viable for the future MOS technology shows Frenkel-Poole conduction at medium oxide fields of 5-7 MV/cm due to presence of bulk traps and Fowler-Nordheim conduction at high oxide fields greater than $8 \mathrm{MV} / \mathrm{cm}[8,9]$. Presently, the high-K oxides are grown by a wide variety of processing techniques such as e-beam evaporation, jet vapor deposition, atomic layer deposition, ionbeam sputtering, pulsed-laser deposition etc., and these oxides may exhibit varied conduction mechanisms before and after annealing. Ultimately, the objective is to remove the bulk traps by improved processing techniques to make the films amorphous and exhibit only FN tunneling as in thermal $\mathrm{SiO}_{2}$ or JVD nitride. However, the conduction and valence band offsets and the carrier masses presented in Table II can help to simulate FN tunneling currents at high field region and estimate the breakdown strength of the films, or alternatively, a given FN tunneling current-voltage characteristics at high fields on a high-K oxide film can be utilized to calculate the band offsets, bandgap and carrier mass values by the present technique. It is interesting to note at this point that the FN technique of MIS characterization is applicable to both the high-K oxides having direct tunneling equivalent oxide thickness of less than $4 \mathrm{~nm}$ and the oxide containing some bulk traps. This is because the FN tunneling is exhibited by both types of oxides at high oxide electric fields.

The electron and hole effective mass of $0.42 \mathrm{~m}$ and $0.58 \mathrm{~m}$ of the thermal $\mathrm{SiO}_{2}$ is the same for thicknesses ranging from $8.5 \mathrm{~nm}$ to $1 \mathrm{~nm}$ as presented in the Table II. Earlier determination of electron effective mass of $0.42 \mathrm{~m}$ for a $100 \mathrm{~nm}$ thermal oxide [3] is also the same as those in the Table II for thinner $\mathrm{SiO}_{2}$ films. The report of hole effective mass of $0.58 \mathrm{~m}$ in $40 \mathrm{~nm} \mathrm{SiO}{ }_{2}$ grown on $4 \mathrm{H}-\mathrm{SiC}$ [6] provides another supporting evidence of the above values. Thus it can be safely concluded that for all thicknesses of the $\mathrm{SiO}_{2}$ in which bulk silica characteristics are present, the electron and hole effective masses are the same as above.

The obtained electron and hole effective mass values of $0.42 \mathrm{~m}$ and $0.58 \mathrm{~m}$ in $\mathrm{SiO}_{2}$ are for a free Fermi gas model of carriers at the emitting electrode. However, if quantum confinement of carriers is considered at the emitting electrode, where the conduction or valence band edge forms a triangular potential well when biased, then these values have to be corrected. Nearly $70 \%$ population of carriers is calculated to be residing at the ground state subband energy level in the potential well [18], which reduces the electron barrier to oxide conduction band by $0.2 \mathrm{eV}$ [4], and the hole barrier to oxide valence band by $0.16 \mathrm{eV}$ [19] in $\mathrm{Si}<100>\mathrm{MOS}$ devices. The corrected electron and hole barrier heights due to quantum confinement results in the electron and hole effective masses in the thermal oxide to $0.51 \mathrm{~m}$ and $0.65 \mathrm{~m}$ respectively. It needs to be mentioned here that quantum confinement which causes bandgap broadening at the semiconductor-oxide interface and affects the carrier effective mass calculations, is valid for extremely thin channels in the MOSFETs of a few tens of angstroms. The peak of the electron concentration lie several angstroms away from the $\mathrm{Si} / \mathrm{SiO}_{2}$ interface because the nature of the wave function of the ground state where most of the carriers reside is a half sine wave.

\section{Conclusion}

The present study demonstrates that a unique set of band offsets, unknown bandgap of the oxide, and the carrier masses in the oxide can be calculated from only the FN tunneling slope constant values obtained from MOS devices on silicon. For the $\mathrm{SiO}_{2} / \mathrm{Si}<100>$ MIS structure, the conduction band offset is $3.2 \mathrm{eV}$, the valence band offset is $4.6 \mathrm{eV}$, the $\mathrm{SiO}_{2}$ bandgap is $8.9 \mathrm{eV}$, and the electron and hole effective masses in $\mathrm{SiO}_{2}$ are $0.42 \mathrm{~m}$ and $0.58 \mathrm{~m}$ respectively. If the carriers are quantum confined at $\mathrm{Si} / \mathrm{SiO}_{2}$ interface, then the electron and hole effective mass values become $0.51 \mathrm{~m}$ and $0.65 \mathrm{~m}$ respectively. The electron and hole effective masses in thermal $\mathrm{SiO}_{2}$ are the same for all thickness of oxide. The FN technique called BOEMDET described in the present study can be utilized to characterize an MIS structure. Once characterized, the parameters can be used to simulate the current-voltage characteristics and estimate the dielectric breakdown fields. Also, the characteristics of two different MIS structures can be compared to give insight into relative reliability.

\section{References}

[1]. J. Robertson, "High dielectric constant oxides", Eur. Phys. J. Appl. Phys., vol. 28, pp.265-291, Dec. 2004.

[2]. G.D. Wilk, R.M. Wallace, J.M. Anthony, "High-K gate dielectrics: Current status and materials properties considerations", J. Appl. Phys, vol.89, pp.5243-5275, May 2001

[3]. M. Lenzlinger and E.H. Snow, "Fowler-Nordheim tunneling into thermally grown $\mathrm{SiO}_{2}$ ", J. of Applied Physics, vol. 40, pp. 278283, Jan. 1969.

[4]. Z.A. Weinberg, “On tunneling in metal-oxide-silicon structures”, J. of Applied Physics, vol. 53, pp. 5052-5056, Jul. 1982.

[5]. R.K. Chanana, K. McDonald, M. Di Ventra, S.T. Pantelides, L.C. Feldman, G.Y. Chung, C.C. Tin, J.R. Williams, R.A.Weller, "Fowler-Nordheim hole tunneling in p-SiC/SiO 2 structures", Appl. Phys. Lett., vol. 77, pp. 2560-2562, Oct. 2000.

[6]. R.K. Chanana, "Determination of hole effective mass in $\mathrm{SiO}_{2}$ and $\mathrm{SiC}$ conduction band offset using Fowler-Nordheim tunneling characteristics across metal-oxide-semiconductor structures after applying oxide field corrections", J. of Applied Physics, vol. 109, pp. $104508-1$ to -6 , May 2011. 
[7]. R.K. Chanana, "Determination of electron and hole effective masses in thermal oxide utilizing an n-channel silicon MOSFET", IOSR-Journal of Applied Physics, vol. 6, Issue-3, pp.1-7, May-June, 2014

[8]. Joo-Hyung, V. A. Ignatova, P. Kucher, M. Weisheit, E. Zschech, " Post annealing effect on ultra-thin Hf-based high-k gate oxides on Si”, Current Applied Science, vol.9, pp.104-07, March 2009.

[9]. W.J. Zhu, T.P. Ma, T. Tamagawa, J. Kim and Y. Di, "Current transport in metal/Hafnium oxide/silicon structure", IEEE EDL, vol. 23, no.2, pp.97-99, February 2002.

[10]. P. Srinivasan, N.A. Chowdhury, and D. Misra, "Charge trapping in ultrathin Hafnium silicate/metal gate stacks", IEEE EDL, vol. 26, no. 12, pp. 913-15, December, 2005.

[11]. T. P. Ma, "Making silicon nitride film a viable gate dielectric", IEEE Trans. On ED, vol.45, no.3, pp.680-690, March 1998.

[12]. Yee Chia Yeo, Qiang Lu, Wen Chin Lee, Tsu-Jae King, C. Hu, Xiewen Wang, Xin Guo, and T.P. Ma, “ Direct tunneling gate leakage current in transistors with ultrathin silicon nitride gate dielectric", IEEE EDL, vol, 21, no.11, pp.540-42, November 2000.

[13]. I.D.Parker, "Carrier tunneling and device characteristics in polymer light-emitting diodes", J.Appl. Phys., vol. 75, pp. 1656-1666, Feb. 1994

[14]. E. Bersch, S. Rangan, R.A. Bartynski, E. Garfunkel, E. Vescovo, "Band offsets of ultrathin high-K oxide films with Si", Physical Review B, vol. 78, pp.085114-1 to 10, 2008.

[15]. S.M. Sze, "Energy Bands and Carrier Concentration", in Semiconductor Devices, Physics and Technology, New York, John Wiley and Sons, 1985, pp. 18-19.

[16]. B. Eitan and A. Kolodny, "Two components of tunneling current in metal-oxide-semiconductor structures", Appl. Phys. Lett., vol. 43, pp. 106-108, Jul. 1983.

[17]. J.W. Keister, J.E. Rowe, J.J. Kolodziej, H. Niimi, T.E. Madey, G. Lucovsky, "Band offsets for ultrathin $\mathrm{SiO}_{2}$ and $\mathrm{Si}_{3} \mathrm{~N}_{4}$ films on $\mathrm{Si}(111)$ and $\mathrm{Si}(100)$ from photoemission spectroscopy", J. Vac. Sci. Technol. B. 17, pp.1831-1835, Jul/Aug 1999.

[18]. Y.T. Hou, M.F. Li, Y. Jin, and W.H. Lai, "Direct tunneling hole currents through ultrathin gate oxides in metal-oxidesemiconductor devices”, J. of Applied Physics, vol. 91, pp. 258-264, Jan. 2002.

[19]. S. Rodriguez, J.A. Lopez-Villanueva, I. Melchor and J.E. Carceller, "Hole confinement and energy subbands in silicon inversion layer using the effective mass theory”, J.Appl. Phys., vol. 86, pp. 438-444, Jul. 1999. 